\title{
The Luna experiment
}

\author{
Carlo Broggini ${ }^{1, a}$ for the LUNA Collaboration \\ ${ }^{1}$ INFN Sezione di Padova, via Marzolo 8, I-35131
}

\begin{abstract}
One of the main ingredients of nuclear astrophysics is the cross section of the thermonuclear reactions which power the stars and synthesize the chemical elements in the Universe. Deep underground in the Gran Sasso Laboratory the cross section of the key reactions of the proton-proton chain and of the Carbon-Nitrogen-Oxygen (CNO) cycle have been measured right down to the energies of astrophysical interest. The main results obtained during the solar phase of LUNA are reviewed before describing the current LUNA program devoted to the study of the nucleosynthesis of the light elements in AGB stars and Classical Novae. Finally, the future of LUNA with the new 3.5 MV accelerator devoted to the study of helium and carbon burning is discussed.
\end{abstract}

\section{Introduction}

Only hydrogen, helium and lithium were synthesized in the first minutes after the big-bang. All the other elements in the Universe are produced by the thermonuclear reactions taking place inside the cauldrons active in the cosmos, i.e. the stars. Nuclear astrophysics studies the reactions which transmute the chemical elements and provide energy to the stars. The reactions occur in the hot plasma of a star, with temperatures in the range of tens to hundreds of millions degrees, inside an energy window, the Gamow peak, which is far below the Coulomb energy arising from the repulsion between nuclei. In this region the cross section is given by:

$$
\sigma(E)=\frac{S(E)}{E} \exp (-2 \pi \eta)
$$

where $S(E)$ is the astrophysical factor (which contains the nuclear physics information) and $\eta$ is given by $2 \pi \eta=31.29 Z_{1} Z_{2}(\mu / E)^{1 / 2}$. $Z_{1}$ and $Z_{2}$ are the nuclear charges of the interacting particles, $\mu$ is the reduced mass (in units of amu), and $E$ is the center of mass energy (in units of $\mathrm{keV}$ ).

At low energies the cross sections are extremely small. Such smallness makes the star life-time of the length we observe, but it also makes the direct measurement impossible in the laboratory. LUNA, Laboratory for Underground Nuclear Astrophysics, started twenty five years ago to run nuclear physics experiments in an extremely low-background environment, the Gran Sasso Laboratory (LNGS), to reproduce in the laboratory what Nature makes inside the stars $[1,2]$.

ae-mail: broggini@pd.infn.it 


\section{Accelerators under Gran Sasso}

Two electrostatic accelerators able to deliver hydrogen or helium beams have been installed in LUNA: first a compact $50 \mathrm{kV}$ home made machine and then, in the year 2000, a commercial $400 \mathrm{kV}$ one. Common features of the two accelerators are the high beam current, the long term stability and the precise beam energy determination. In particular, the $400 \mathrm{kV}$ accelerator can provide up to $0.5 \mathrm{~mA}$ of hydrogen and $0.25 \mathrm{~mA}$ of helium at the target stations, with $0.3 \mathrm{keV}$ accuracy on the beam energy, $100 \mathrm{eV}$ energy spread, and $5 \mathrm{eV}$ per hour long-term stability.

The dolomite rock of Gran Sasso provides a natural shielding equivalent to at least 3800 meters of water which reduces the muon and neutron fluxes by a factor $10^{6}$ and $10^{3}$, respectively. The neutron flux underground is mainly due to $(\alpha, \mathrm{n})$ reactions in the rock, with the alpha particles coming from the ${ }^{238} \mathrm{U}$ and ${ }^{232} \mathrm{Th}$ decay chains. Finally, the activity due to Radon from the rock is suppressed down to the level of few tens of Bequerel $/ \mathrm{m}^{3}$ thanks to frequent air volume exchanges.

\section{LUNA and the Sun}

The first phase of LUNA, the solar phase, has been driven by the solar neutrino problem [3]. In particular, the initial activity has been focused on the ${ }^{3} \mathrm{He}\left({ }^{3} \mathrm{He}, 2 \mathrm{p}\right){ }^{4} \mathrm{He}$ cross section measurement within the solar Gamow peak (15-27 keV). Such a reaction is a key one of the hydrogen burning proton-proton chain, which is responsible for more than $99 \%$ of the solar luminosity. Fig.1 shows the results from LUNA [4] together with higher energy measurements [5-7]. For the first time a nuclear reaction has been measured in the laboratory at the energy occurring in a star. In particular, at the lowest energy of $16.5 \mathrm{keV}$ the cross section is 0.02 pbarn, which corresponds to a rate of about 2 events/month, rather low even for the "silent" experiments of underground physics. No narrow resonance has been found and, as a consequence, the astrophysical solution of the ${ }^{8} \mathrm{~B}$ and ${ }^{7} \mathrm{Be}$ solar neutrino problem based on its existence has been definitely ruled out. ${ }^{3} \mathrm{He}(\alpha, \gamma){ }^{7} \mathrm{Be}$, the competing reaction for ${ }^{3} \mathrm{He}$ burning, has also been measured by LUNA both by detecting the prompt $\gamma$ rays and by counting the ${ }^{7} \mathrm{Be}$ nuclei from their decay.

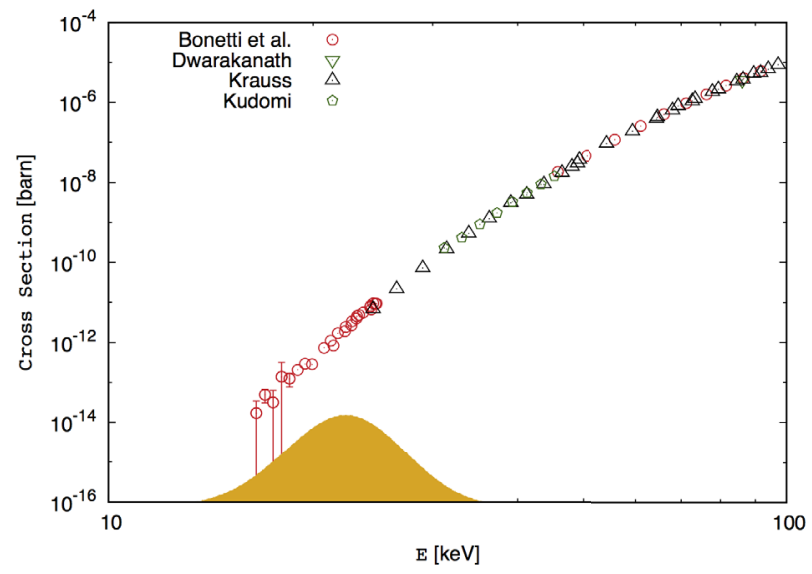

Figure 1. The cross section of ${ }^{3} \mathrm{He}\left({ }^{3} \mathrm{He}, 2 \mathrm{p}\right)^{4} \mathrm{He}$ as function of energy. 
The CNO cycle is responsible for the remaining $1 \%$ of the solar luminosity. ${ }^{14} \mathrm{~N}(\mathrm{p}, \gamma){ }^{15} \mathrm{O}$ is the slowest reaction of the cycle. In particular, it is the key reaction to predict the ${ }^{13} \mathrm{~N}$ and ${ }^{15} \mathrm{O}$ solar neutrino flux, which depends almost linearly on its cross section. The results obtained first with the germanium detector [8], then with the BGO set-up [9] and, finally, with a composite germanium detector [10] were about a factor two lower than the existing extrapolation from previous data at very low energy (with a total error now reduced to 8\%). Because of this reduction the CNO neutrino yield in the Sun is decreased by about a factor of two. Thanks to the relatively small error on the cross section, it will be possible in the near future to measure the carbon and nitrogen content of the Sun core by comparing the predicted CNO neutrino flux with the measured one.

\section{AGB stars and Classical Novae}

A rich program of nuclear astrophysics mainly devoted to $\mathrm{CNO}, \mathrm{Ne}-\mathrm{Na}$ and $\mathrm{Mg}-\mathrm{Al}$ cycles started a few years ago after the solar phase of LUNA. Of particular interest are those bridge reactions which are connecting one cycle to the next, as ${ }^{15} \mathrm{~N}(\mathrm{p}, \gamma){ }^{16} \mathrm{O}[11]$ and ${ }^{17} \mathrm{O}(\mathrm{p}, \gamma){ }^{18} \mathrm{~F}$ [12], the latter competing with ${ }^{17} \mathrm{O}(\mathrm{p}, \alpha){ }^{14} \mathrm{~N}$ [13], or which are key ingredients of gamma astronomy, as ${ }^{25} \mathrm{Mg}(\mathrm{p}, \gamma){ }^{26} \mathrm{Al}$ [14].

Due to the higher Coulomb barrier of the reactions involved, the cycles become important at temperatures higher than the one of our Sun, i.e. during hydrogen burning in the shell of AGB stars and during the thermonuclear runaway of classical Novae (about 30-100, and 100-400 million degrees, respectively). Relatively unimportant for energy generation, these cycles are essential for the cooking of the light nuclei up to ${ }^{27} \mathrm{Al}$. In particular, LUNA is now measuring ${ }^{22} \mathrm{Ne}(\mathrm{p}, \gamma)^{23} \mathrm{Na}$ [15], the reaction of the Ne-Na cycle with the highest uncertainty, and ${ }^{23} \mathrm{Na}(\mathrm{p}, \gamma)^{24} \mathrm{Mg}$, the reaction connecting the $\mathrm{Ne}-$ $\mathrm{Na}$ cycle to the $\mathrm{Mg}-\mathrm{Al}$ one.

\section{What next: helium and carbon burning}

After hydrogen burning the natural evolution of LUNA is the study of the next steps in the fusion chain towards ${ }^{56} \mathrm{Fe}$ : helium and carbon burning. In particular, ${ }^{12} \mathrm{C}(\alpha, \gamma){ }^{16} \mathrm{O}$ determines the abundance ratio between carbon and oxygen, the two key elements to the development of life, and it shapes the nucleosynthesis in massive stars affecting the outcomes of both thermonuclear and core-collapse supernovae.

Equally important are ${ }^{13} \mathrm{C}(\alpha, \mathrm{n}){ }^{16} \mathrm{O}$ and ${ }^{22} \mathrm{Ne}(\alpha, \mathrm{n})^{25} \mathrm{Mg}$, the sources of the neutrons which synthesize half of the trans-iron elements through the S-process: neutron capture followed by $\beta$ decay. Finally, the ${ }^{12} \mathrm{C}+{ }^{12} \mathrm{C}$ fusion reaction is switching on the carbon burning. Its rate determines the evolution of a massive star up to a slowly cooling white dwarf or up to a core-collapse supernova. It also affects the ignition conditions and time scales of thermonuclear supernovae, the standard candles of Cosmology.

This program requires a new 3.5 MV accelerator which is going to be installed underground in the north side of Hall B of Gran Sasso in spring 2018. The accelerator room (fig.2) will have 80cm thick concrete walls and ceiling working as neutron shielding. The room will host a single-ended electrostatic accelerator, with two beam lines (only one of them fed at a time) able to deliver $\mathrm{H}, \mathrm{He}^{+}$, $\mathrm{C}^{+}$and $\mathrm{C}^{2+}$ beams at high current (from $1 \mathrm{~mA}$ of $\mathrm{H}$ to $100 \mathrm{e} \mu \mathrm{A}$ of $\mathrm{C}^{2+}$ ). After the underground mounting of the accelerator and its commissioning, the first physics run will start at the beginning of 2019. 

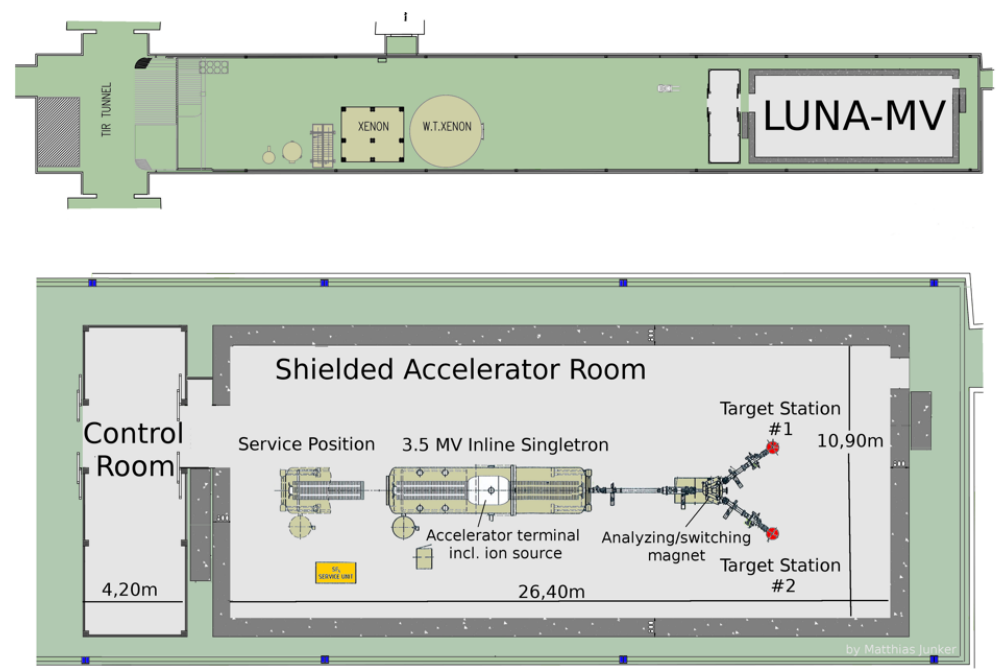

Figure 2. The LUNA-MV facility in the north side of Hall B at LNGS and the 3.5 MV accelerator.

\section{Conclusions}

Underground nuclear astrophysics was born twenty five years ago in the core of Gran Sasso, with the aim of measuring cross sections at very low energy. After the solar phase, LUNA is now studying the hydrogen burning reactions which are responsible for the cooking of the light elements in AGB stars and classical Novae. The future of LUNA is going to start with the installation of a new $3.5 \mathrm{MV}$ accelerator in spring 2018 and it will be focused on the study of helium and carbon burning in stars.

\section{References}

[1] H. Costantini et al, Rep. Prog. Phys. 72, 086301 (2009).

[2] C. Broggini et al, Annu. Rev. Nucl. Part. Sci. 60, 53 (2010).

[3] G. Bellini, C. Broggini, A. Guglielmetti, Eur. Phys. J. A 52, 88 (2016).

[4] R. Bonetti et al, Phys. Rev. Lett. 82, 5205 (1999).

[5] A. Krauss et al, Nucl. Phys. A467, 273 (1987).

[6] M. Dwarakanath and H. Winkler, Phys. Rev. C 4, 1532 (1971).

[7] N. Kudomi et al, Phys. Rev. C 69, 015802 (2004).

[8] C. Formicola et al, Phys. Lett. B 591, 61 (2004).

[9] A. Lemut et al, Phys. Lett. B 634, 483 (2006).

[10] M. Marta et al, Phys. Rev. C 78, 022802 (2008).

[11] A. Caciolli et al, Astronomy and Astrophysics 533, A66 (2011).

[12] D.A. Scott et al, Phys. Rev. Lett. 109, 202501 (2012).

[13] C.G. Bruno et al, Phys. Rev. Lett. 117, 142502 (2016).

[14] O. Straniero et al, Astrophysical Journal 763, 100 (2013).

[15] F. Cavanna et al, Phys. Rev. Lett. 115, 252501 (2015). 